\title{
Medical Healthcare System Based on Wireless Body Area Networks: The Importance of Anomaly Detection
}

\author{
Hayder Jassim Hassaballah, Rashid Ali, Bushra Abdulkareem Abdulazeez Al- \\ Hayali
}

\begin{abstract}
In this research paper, a modern framework is presented to detect anomaly in medical wireless body sensor network systems that are incorporated in distant observation of patient's vital signs. The suggested framework effects analysis of data in a sequential manner using a mini gateway utilized as a root station to discover abnormal alterations and to deal with inaccurate computations in gathered medical information minus advance awareness of irregular occurrences or consistent data patterns. The suggested perspective relies on Principal Component Analysis (PCA) utilized in spatial analysis and dimension reduction for gathered medical details. The key goal is distinguishing defective calculations from clinical dangers for reduction of false alarms prompted by incorrect computations or ill-behaved sensors. The result from the experiments on real medical datasets reveal that the suggested PCA perspective is able to attain good discovery perfection with lesser falt alarm rate when contrasted with other perspectives that fail to minimize the excessive dimension of gathered more information so in multivariate Wireless Body Sensor Networks (WBSN) implementations, and dynamic streaming nature of sensor data, mostly in medical implementations.
\end{abstract}

Keywords: WSN, WBSN, Anomaly Detection, Dimension Reduction, PCA.

\section{INTRODUCTION}

Wireless sensor networks (WSNs) are networks of tiny, low cost, low energy, and multifunctional sensors which are densely deployed to monitor a phenomenon, track an object, or control a process [1, 2]. Currently, WSNs are used in many application domains, which include: personal applications such as home automation; business applications such as sales tracking; industrial applications such as architectural and control. In addition,for health care applications such as medical and non-medical [3]. A new concept that seems to be

Revised Manuscript Received on February 15, 2020.

* Correspondence Author

Hayder Jassim Hassaballah*, Middle Technical University, Electrical Engineering Technical College, Department of Medical Instrumentation Techniques Engineering/ / Iraq. E-mail: hayderalmusawy@gmail.com,

Rashid Ali, Middle Technical University, Electrical Engineering Technical College, Department of Medical Instrumentation Techniques Engineering/ / Iraq. E-mail: rashidfayadh47@gmail.com,

Bushra Abdulkareem Abdulazeez Al- Hayali, Middle Technical University / Institute of Medical Technology Baghdad / Iraq. E-mail: Bushra.a_azeez@yahoo.com

(c) The Authors. Published by Blue Eyes Intelligence Engineering and Sciences Publication (BEIESP). This is an open access article under the CC BY-NC-ND license (http://creativecommons.org/licenses/by-nc-nd/4.0/) the future of WSNs is the "Internet of Things" (IoT) which expects that each object in human life is equipped with sensors that communicate with each other to constitute a network that make life much easier [4]. WSNs have been approached from different disciplines, including networking, embedded systems, information processing, distributed systems and signal processing. As a result, variety of research areas have been evolved including routing protocols, localization strategies, sensing hardware design, query processing, data mining, information processing, security and privacy.

Sensor data analysis is of high importance to decision makers. It was reported by Zhang [5], that the purpose of using WSN is not only to collect data from the field of deployment but more importantly the analysis of this data at timely manner that leads for making some important decisions. Therefore, the data quality is the main concern since it reflects the real state of monitored environments.

Unfortunately, the raw measurements collected by sensor nodes suffer from inaccuracy and incompleteness [6]. These inaccurate sensor measurements which are known as anomalies may be caused by reasons related to the sensor device itself or sensing environment. Resource constraints of sensor devices in terms of storage, energy, processing, and bandwidth may contribute to nodes failures and therefore reporting of anomalous readings. malicious attacks such as a denial of service, black hole, selective forwarding, and wormhole attacks are the other reasons which contribute to generate such inaccurate and low-quality data.

The inaccurate or incomplete data measurements caused by reasons are known as anomalies. Anomaly is defined by Hodge and Austin [7], as "an observation that seems to be inconsistent with the rest of dataset".

This study evaluates the medical health care implementations of wireless sensing systems and utilizes WBSN instead of WSN. Also, In this paper, we aim to accurately identify abnormal measurements in the data gathered by medical WSNs. We consider a scenario where many sensors are attached to the patient, in order to monitor some physiological parameters, and transmit the data to a smart phone which must analyze the collected data, and raise alarms to the caregiver only when the patient health degrades. We seek to detect and to remove outliers in order to reduce false alarms triggered by inconsistent sensor readings which significantly deviate from the normal data measurements. The objective is to raise alarms only when the patient's health is abnormal (illness).

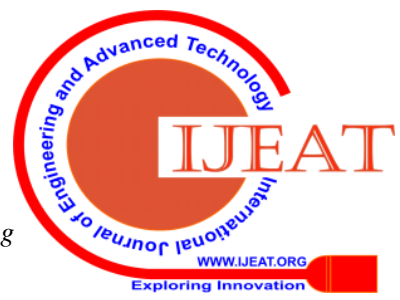


The other parts of the paper are ordered as stated. The second section examines the background of WBSN. The third section explains the suggested procedure for anomaly detection structure. The fourth part brings out the outcomes to illustrate how effective the suggested technique is. Lastly, the conclusion is presented in fifth part of this paper.

\section{WIRELESS BODY SENSOR NETWORKS (WBSNS) / BACKGROUND}

\section{A. Applications of WBSN}

WBANs aid various technologies and fascinating innovations. The innovations are inclusive of areas like smart health care, facilitated elderly livelihood, disaster reply and interactive gaming. Classification of WBAN innovations from literature fall into medical and non-medical innovations [3]. Researchers in [8] contrasted between in-body and on-body innovations, for medical ones. This section discusses the key classifications of medical applications.

\section{- Telemedicine and remote patient monitoring.}

The increase costs of healthcare plus the maturity of the world inhabitants are a contribution to the developments in telemedicine network for dispatch of various services in healthcare. Telemedicine allows local distribution of victim care through integrated health information structures and telecommunications applications and permits scientists, doctors and other medical executives over the globe to assist extra victims. Actually, as a result of the provided indications from the body sensors, collected details get efficiently managed trust worth and precise physiological approximations by further permitting remote physician to possess immediate suggestions for medical discovery and instruction. The mentioned smart health care structures can furnish with systems for detection processes, support of terrible diagnostics and supervised healing from a surgical process. Patient examination systems basically manage essential signs, including provision of actual feedback and data to aid patient recovery [9]. These circumstances mean the victim can be kept under a physician control in natural physiological condition minus restricting their daily undertakings and minus causing him high cost injury. Day-to-day undertaking control examines the undertakings in the everyday life of a victim suffering from particular illness; on the contrary in hospital control centers on victims staying within the hospital for intensive care and monitoring, or occasionally extended duration. Meantime, various medical variables are constantly documented. Post-surgery in-home recuperation control handles victims in their post recovery duration succeeding a medical surgery plus a recuperation duration in a medical facility. A WBAN furnishes constant variables of the physiological indicators and permits improved disclosure organ collapses and quick diagnose of critical circumstances. These remote-control structures will be secure, suitable and cost effective. This field has had various suggestions from past works. Few attempted to depict a universal framework for aiding many cases yet others attempted to examine certain illnesses [10]. Cardiovascular diseases, diabetes, cancer detection, asthma, Alzheimer and artificial retina are few instances of certain remote patient monitoring implementations [11] [12].

\section{B. Rehabilitation and therapy}

The objective of rehabilitation is permitting victims to regain their functional ability to normal via proper rehabilitative therapies preceding hospital dismissal [13] Rehabilitation is a vigorous procedure utilizing provided amenities for correction of any excluded motion action to attain a projection. There needs to be constant monitoring and rectification to provide regain of health to a previous stroke victim during a rehabilitation procedure, to achieve expected motion patten. Accordingly, discovering human motion is important and a necessity in a home-based rehabilitation strategy. Examples of elements that provide specificity in rehabilitation as a study area with exact limitations and necessities include sensor diversification, multi-sensor data fusion, real-time feedback for patients and virtual reality integration [14].

\section{- Biofeedback}

There is possibility of self-remote tracking of human body via WBANs for gathered data access by sensors. Implantation of sensors is done in people for monitoring behavior or pathologies to assist victims have good health via biofeedback events like analyzing temperature, detecting pressure in blood, Electrocardiography (ECG), Electromyography (EMG), among others. This study explains biofeedback as computation of physiological undertakings inclusive of various prospective important variables and feeding them back to a client by enabling control and modification of his physiological undertakings for further health and conduct improvement [15].Incorporation of biofeedback has been in place since 1960s and brings in usefulness in management of emotional conditions and involuntary anatomy tasks like migraine and blood pressure. Biofeedback gadgets are inclusive of those that track breathing, heart function, muscle activity and brainwaves [15].

\section{- Ambient Assisted Living}

For enlargement of technology-assistive living innovations to aid in safety and independent maturity, the aging populace, rise in formal healthcare cost and the benefit placed by people on living independently are of benefit. Accomplishments in this area enhance life quality for maintenance of a more independent habit through home automation [16]. Actually, the emergence of assisted living as a choice for persons with disability and mature beings who are regarded dependent but do not require all time medical care like nursing and retirement facilities.

An ambient senor system can discern and manage the variables of the living surroundings and further furnish the body to the main area due to constant cognitive and physical tracking. Estimation of the health situation of these persons is possible via the rate of heart beat, blood pressure and accelerometer data. Connection of the system to a hospital is possible for further examination and crisis intervention if there are vigorous alterations in the monitored variables for changes from ordinary stretch [17]. 


\section{Security of WBSN.}

Based on the differences from other networks and due to the sensitive environment in where they are deployed, WBSNs are susceptible and vulnerable to many kinds of security breaches either from inside or outside the medical network. It is also clear that most of security solutions adopted for other networks cannot be directly ported for WBSN due to some technical reasons as described in Akyildiz et al. [18].

To protect WBSNs against different kinds of vulnerabilities, prevention-based (e.g. cryptography and authentication) and detection-based (e.g. Intrusion Detection) mechanisms are put in place.

Prevention based security mechanisms for WBSN constitute the main defense line against security penetrations. These mechanisms include cryptography, key management, secure routing, and data aggregation. The selection of cryptographic techniques should be made carefully to meet the constraints of sensor nodes. These cryptographic techniques are evaluated using some special metrics like size of code, and computation overhead. While Intrusion Detection Systems (IDS) is considered as the second line of defense against network attacks that preventive mechanisms fail to address. An IDS is defined by Hossain, [19] A system that dynamically monitors the events taking place in a system, and decides whether these events are symptoms of an attack or constitute a legitimate use of the system". However, there are many challenges posed against the application of IDS for WBSNs. These challenges are due to the lack of resources like energy, processing, and storage. In general, IDS schemes are categorized into misuse and anomaly, anomaly IDS schemes can be classified into supervised based and unsupervised based IDS. The supervised anomaly-based intrusion detection schemes involve training the detection model which requires prior knowledge about what is normal behavior, and what is anomalous. Meanwhile, the unsupervised scheme does not require any prior knowledge to build the detection model; instead, it uses some measures to decide if the data instance is normal or anomalous [20].

Anomaly detection based on IDS using unsupervised machine learning has been used due to the dynamic and changeable characteristics of medical vital signs of the body. Many challenges of anomaly detection in WBSN will be explained in the next section.

\section{Challenges of Anomaly Detection in WBSN}

The main challenge for anomaly detection in WBSN is how to achieve high detection effectiveness with minimum energy cost. Many anomaly detection solutions have been proposed in the literature for traditional network systems. However, the nature of sensor data and the context of WBSN make these solutions not suitable for WBSN. The challenges that should be considered during the design of suitable anomaly detection solutions for WSN are summarized in the following paragraphs [21].

\section{A. Resource Limitations}

WSNs are made up of cheap sensors which are very resource constrained in terms of memory and processing. Therefore, the design of any anomaly detection solution for WSN should consider the minimization of network resources utilization especially in processing and storage.

\section{B. Dynamic Streaming Data}

The dynamic streaming nature of sensing data is another challenge. Generally, there is no prior knowledge available to build the normal distribution of sensing data (normal reference model). Even if this knowledge is available in a specific point of time, it is insufficient for the future because of the dynamic streaming that may change the nature of distribution over the time.

\section{High Dimensional Data}

Besides the possible increase of network size, the dimension of collected data may also increase. The increase of data dimension will incur higher computational complexity that drains the energy and memory of sensors. Therefore, data dimensionality reduction is crucial to save the limited WSN resources and hence prolong the lifetime of WSNs.

The main goal of this paper is how to reduce the dimensional reduction of collected data using Principle Component Analysis (PCA). It has been found that PCA is one of the most candidate dimensionality reduction techniques that can be used efficiently in solving the problem of high dimensional data that can be employed for efficient anomaly detection solutions.

\section{PROPOSED METHOD}

\section{A. WBSN deployment scenario}

This study takes into consideration a common medical disposition structure, where $\mathrm{M}(\mathrm{M} \leq \mathrm{p})$ wireless nodes $\left(\mathrm{S}_{1}\right.$, $\ldots, \mathrm{S}_{\mathrm{N}}$ ) with controlled assets are positioned on the victim's body as in Fig. 1. The sensors are employed for gathering crucial signals (heart rate and blood pursue) and transfer the gathered details at uniform time duration to a sink gadget. A compact smart phone is put on the arm of the victim to gather details from sensors. The gathered details are computed immediately on the smart phone for detection of any inconsistency and send alarms for caretakers only during health degradation (failed respiratory, cardiac arrest) of a victim. Defective computations must be discovered and separated for reduction of incorrect alarms and hinder defective detection.

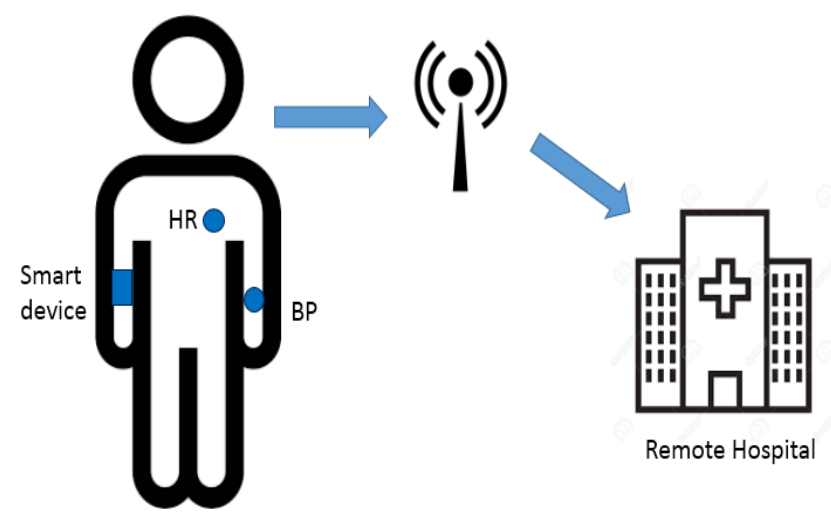

Figure 1 : WBSN deployment scenario 


\section{B. Research Methodology}

This section provides the research framework of implementing the objectives of the present study, the research framework consists of three phases where each phase's output is used as input for the next phase. Figure 2 provides an overview of the research framework.

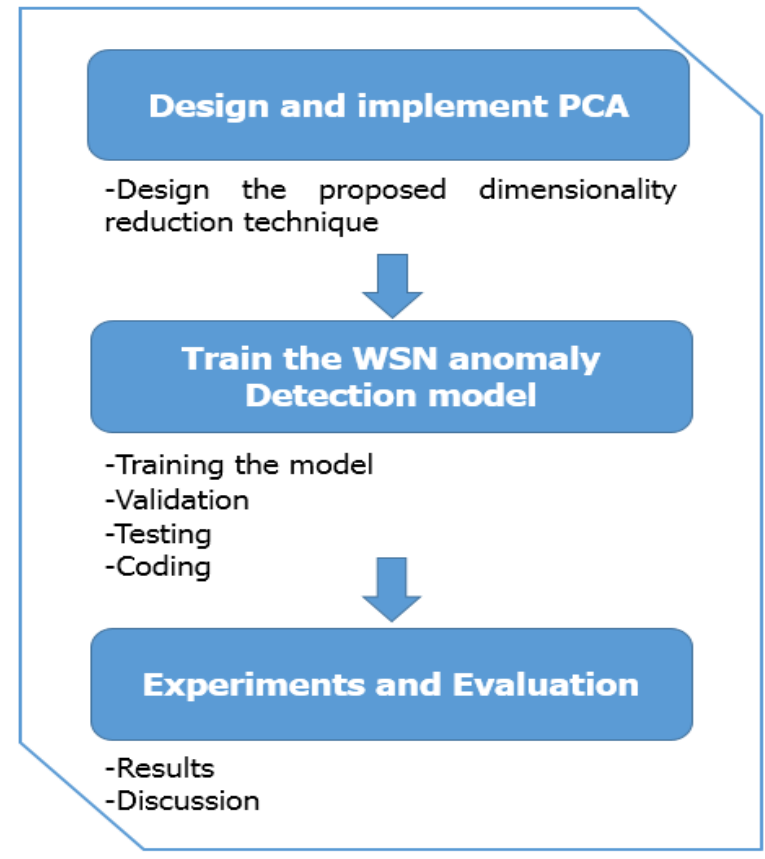

Figure 2: Research framework

To implement the objectives of the proposed study, two stages are involved, namely offline training and online implementation. In the training phase, the Heart rate data and Blood pressure data were collected from measurements. This collected data underwent several pre-processing operations such as standardization, normalization, imputation and noise removal. The reduction process was then carried out using PCA technique to obtain the reduction parameters like eigenvectors and eigenvalues. These parameters are then stored in the node for the purpose of reducing the dimensionality of the incoming measurements observed by that node at implementation phase. They also are stored in the cluster head for approximating the measurements.

In the online implementation phase, PCA carries out the dimensionality reduction on the data observed at real-time using the stored eigenvectors and eigenvalues parameters obtained in the training phase. The reduced data is then sent to cluster head, where the approximation is carried out to restore these data to the original form by same parameters used at the reduction time. The data is recomputed if the change in the environment variables reached a predefined threshold. The outcome of this stage is the reduced data measurements that replace the original data at the detection phase. As such, the computational complexity, communication overhead, and memory utilization were significantly reduced at the real-time deployment of the proposed anomaly detection model. This contributes to improve the efficiency of the proposed anomaly detection model that was trained on such reduced data. Implementation details of PCA technique and its incorporation into the proposed anomaly detection model are elaborated in section 4.

\section{RESULT AND DISCUSSION}

\section{A. The Proposed PCA-Based on Anomaly Detection Model.}

The main reason of quick sensor energy depletion in WBSNs is the data transmission among nodes. The transmission of one-bit data was estimated to consume equivalent power needed to process thousands of bits in sensors. This implies that most of sensor energy is consumed in radio communication rather than data sensing or processing. Therefore, data dimensionality reduction is crucial to minimize the energy consumption in radio communication and hence prolong the lifetime of WBSNs. In this research, the reduced data are utilized by the anomaly detection schemes to further reduce the computational complexity of detection methods.

Two research activities are involved in this study which are pre-processing of the sensor collected data and dimensionality reduction of data. The data is necessary to be pre-processed before it can be fed to any data mining technique. This involves converting the data into suitable format for processing by these techniques. This phase is also aimed at enhancing efficiency of anomaly detection model by reducing dimensionality of data and therefore reducing the power consumption. Two pre-processing procedures were adopted; data transformation and data normalization. Since the features of sensor data are often occurring within different numeric ranges, a suitable data transformation procedure is required to alleviate the effect of that occurrence on the data mining and machine learning techniques. In this study, similar transformation procedure used in related works was adopted. After that each feature was normalized into the range $(0,1)$. It should be noted that, for the centralized scenario, data transformation and the data normalization will be performed at the cluster head for all data. In distributed scenario, each node sends summary of their collected data to the cluster head which includes the number, the linear sum, and the linear sum of squares of their local data vectors. In addition, a vector of the maximum and minimum values of each feature is also sent to the cluster head from each node. Upon receiving this information, cluster heads calculate the global mean, global variance, global max, and global min. These global values are sent back to the sensor nodes to be used to pre-process their local data. A reduced feature set in a dataset can reduce the complexity of anomaly detection by speeding up the detection and improves the detection accuracy.

The networks structure adopted for this paper as shown in Figure 3 is the hierarchical or cluster-based structure. In this structure, the network is grouped into a number of clusters where each cluster has an organizational node called cluster head $(\mathrm{CH})$ which usually has additional 


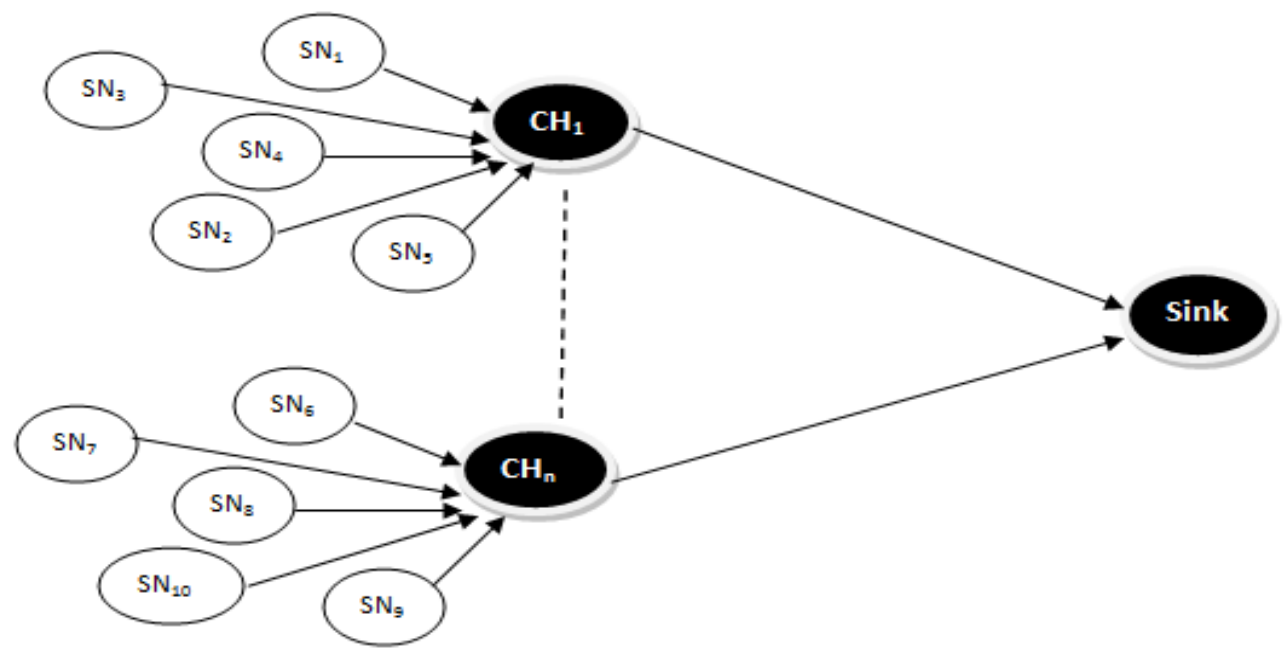

Figure3 Hierarchical sensors structure

\section{B. The design of the proposed PCA-Based Anomaly Detection Model}

The results obtained from applying PCA are the eigenvectors and eigenvalues, which will be then used as features to train the machine learning classifier model. The pseudo code for initialization phase is shown in Figure 4 and the design of the proposed model is shown in Figure 5.

\section{Input: Data collected from sensors \\ Output: The classification of the data as normal or anomalous}

\#\#Training Phase (offline)

1: Collect data from sensors.

2: Do data normalization and Standardization

3: Extract the raw features from data

4: Apply PCA on the raw data and features

5: select the best $n$ eigenvector as PCA features.

6: feed the selected PCA features into logistic regression classifier.

\#\#Testing phase (online):

7: read the new measurement (data) from the sensor

8: apply PCA to newly obtained data

9: Feed the data with PCA features into the classifier

10: The classifier will determine whether the measurement is normal or anomalous.

Figure 4. The pseudocode for the proposed model

\section{Evaluation Metrics.}

To evaluate how effective PCA is for dimensionality reduction, this study used the approximation accuracy and approximation error as they are common metrics widely used by the extant research Carvalho et al., [22]; Le Borgne et al., [23]. In addition, the performance of the proposed WSN's anomaly detection model is measured by several metrics including detection accuracy, detection rate, false positive rates and false negative rates. These measures are well -known and commonly used by the extant research as well
Xie et al. [24] Xie et al., [25], and Zhang et al., [26] Moreover, computational complexity, communication overhead, and memory utilization were used to evaluate the efficiency of the proposed model based on the computation theory as it is commonly practiced by the related works such as Xie et al., [24] Moshtaghi et al., [27], Rajasegarar et al., [28], and Zhang et al.,[29]. Equations (1), (2), (3), and (4) were used to calculate the detection accuracy, detection rate, precision, and the F measure, respectively. 


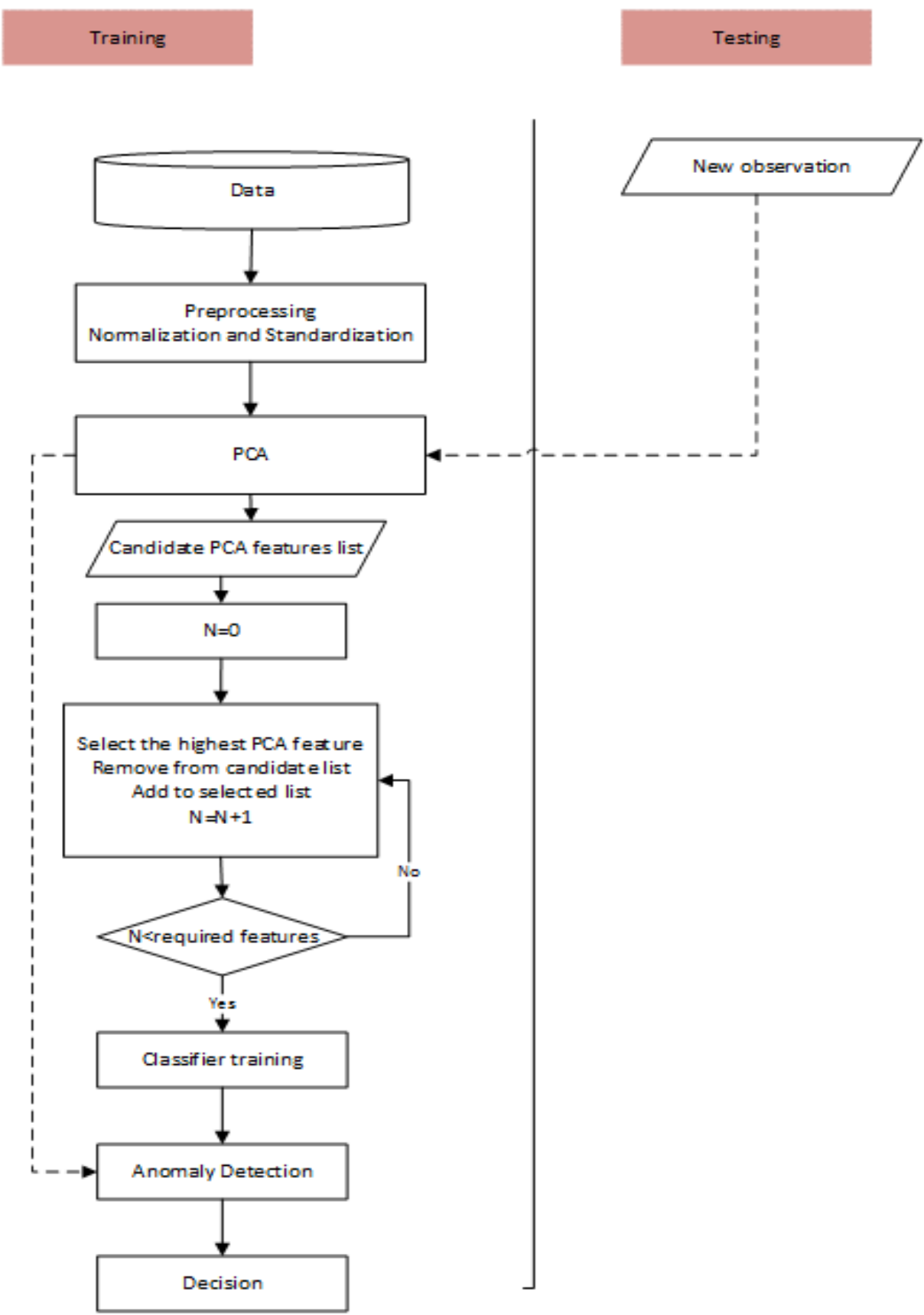

Figure 5. The design of the proposed model

$$
\begin{aligned}
& A C C=\frac{T P+T N}{T P+T N+F P+F N} \\
& D R=\frac{T P}{T P+F N} \\
& \text { Precision }=\frac{T P}{T P+F P}
\end{aligned}
$$$$
F 1=\frac{2 \times \text { Precision } \times \text { Recall }}{\text { Precision }+ \text { Recall }}
$$

where $T P_{y} T N_{s} F P_{y} F N$ denote the true positive, true

negative, false positive and false negative respectively.

\section{Results analysis and Comparison}

Figures 6 and 7 show data distribution of the medical data collected from sensors. As can be seen from figures 6 and 7 the data are not normally distributed. This is attributed to the randomness nature of such data as there are several factors that govern the level of humidity, heart rate and blood presser. These factors are unpredictable. In addition, these three sensors collect relatively correlated data. As such, the randomness in one of these data creates randomness in the other data as well. On the other hand, the data collected by the temperature sensor is normally distributed. This is attributed to the predictability of this measurement and the small range of values that the temperature takes. 


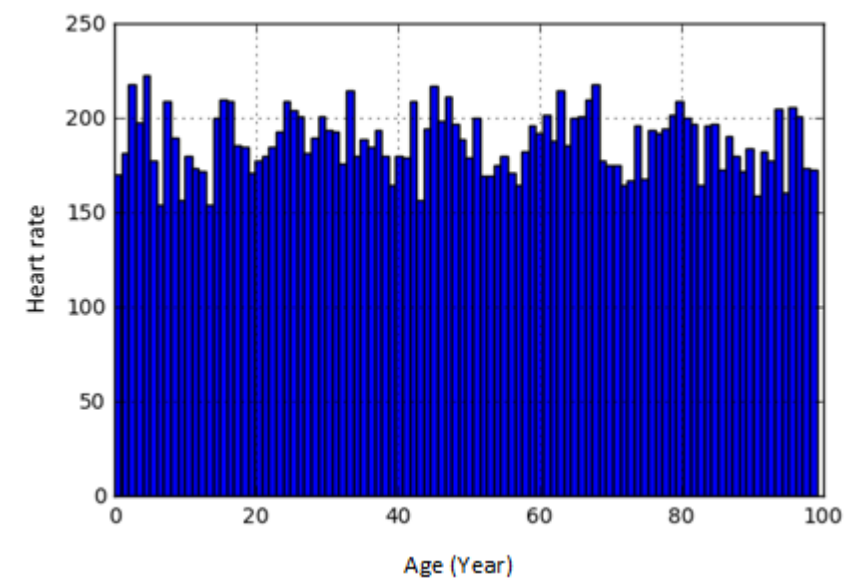

Figure 6. The distribution of the data collected by the heart rate sensor

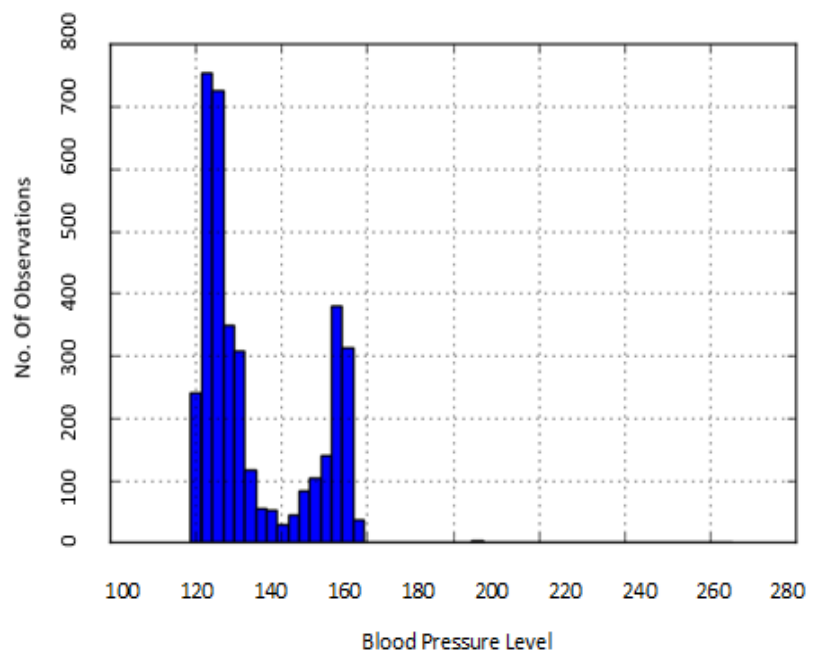

Figure 7 . The distribution of the data collected by the temperature sensor

Figure 8 shows the comparison results of the proposed PCA-Based AD model with the model without involving PCA. As can be seen, the proposed PCA-based model outperformed the model without PCA. That is, the precision, recall, F1 of the proposed model were higher than that of the model without PCA. This is attributed to the ability of PCA technique to reduce the dimensionality of the data and select the most relevant features to be used by the detection model. Such reduction in data dimensionality prevents the overfitting that reduces the detection accuracy. In addition, the accuracy of the detection model was slightly higher than that of the model without PCA. This is attributed to the high false alarms that anomaly detection approach is infamous with. To find out how significant the improvement that the proposed model achieved, the t-test significance test was conducted. As a common practice by the extant research, the significance level was adjusted to the standard value ( $\alpha=0.05$ ) such that the improvement is deemed significant if p-value was less than $\alpha[1,2]$. The conducted t-test reveals that $\mathrm{p}$-value was 0.03 , which is less than the standard value of $\alpha$. As such the improvement that the proposed PCA-Based AD model was significant.

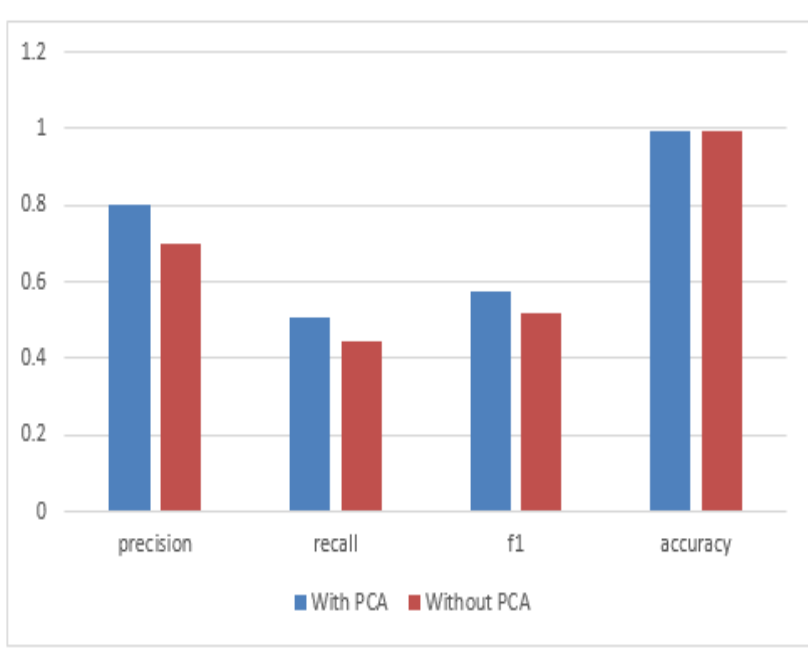
Figure 8. The comparison between the proposed
PCA-Based AD and the model without AD

\section{CONCLUSION}

This paper suggested an unsupervised procedure to detect anomaly in medical WBNSs where incorrect computations and added data could endanger the life of the tracked victim. The suggested technique relies on PCA model to diagnose abnormal computations and to discern incorrect computations from a clinical predicament via incorporation of spatial and temporal connection linking tracked elements. The relevance of the system is kept through upgrading the statistical values and acquiring more accurate examination of the ordinary situation of the victim. The suggested procedure is appropriate for online diagnosis and separation of incorrect computations with less computational difficulty and cache necessities. The technique was examined through actual medical datasets. The investigated outcomes exhibit the efficiency of the suggested technique in lowering the quantity of incorrect alarms emanating from erroneous computations (or harmfully added information) in medical WBSNs.

In most cases, gathered computations are normal. The lessening of transmitted details connecting wireless sensors and sink node are suggested for future incorporation. The next examination will be geared to distributed diagnose of an inconsistency in sensors to lower the misused energy through transfer of erroneous computations.

\section{REFERENCES}

1. Watt, A. J., et al. "Wireless Sensor Networks for monitoring underwater sediment transport." Science of The Total Environment (2019).

2. Khalaf, Osamah Ibrahim, and Bayan Mahdi Sabbar. "An overview on wireless sensor networks and finding optimal location of nodes." Periodicals of Engineering and Natural Sciences 7.3 (2019): 1096-1101.

3. Taha, Mustafa Sabah, et al. "Wireless body area network revisited." International Journal of Engineering \& Technology 7.4 (2018): 3494-3504.

4. Mutlag, Ammar Awad, et al. "Enabling technologies for fog computing in healthcare IoT systems." Future Generation Computer Systems 90 (2019): 62-78. 
5. Zhang, Y. (2010). Observing the Unobservable : Distributed Online Outlier Detection in Wireless Sensor Networks. PhD Thesis.University of Twente, The Netherland.

6. Rassam, Murad A., Anazida Zainal, and Mohd Aizaini Maarof. "Advancements of data anomaly detection research in wireless sensor networks: a survey and open issues." Sensors 13.8 (2013): 10087-10122.

7. Hodge, V., and Austin, J. (2004). A Survey of Outlier Detection Methodologies. Artif. Intell. Rev., 22(2), 85-126.

8. Ullah, Sana, et al. "A comprehensive survey of wireless body area networks." Journal of medical systems 36.3 (2012): 1065-1094.

9. Boulemtafes, Amine, and Nadjib Badache. "Design of Wearable Health Monitoring Systems: An Overview of Techniques and Technologies." mHealth Ecosystems and Social Networks in Healthcare. Springer, Cham, 2016. 79-94.

10. Chakraborty, Chinmay, Bharat Gupta, and Soumya K. Ghosh. "A review on telemedicine-based WBAN framework for patient monitoring." Telemedicine and e-Health 19.8 (2013): 619-626.

11. Nagaraju, V., et al. "Design of miniaturized directional ultra wide band antenna for cancer detection." International Journal of $R F$ Technologies 10.3-4 (2019): 105-113.

12. Karthikeyan, M. V., and J. Martin Leo Manickam. "ECG-Signal Based Secret Key Generation (ESKG) Scheme for WBAN and Hardware Implementation." Wireless Personal Communications 106.4 (2019): 2037-2052.

13. Hadjidj, Abdelkrim, et al. "Wireless sensor networks for rehabilitation applications: Challenges and opportunities." Journal of Network and Computer Applications 36.1 (2013): 1-15.

14. Ali, Nursabillilah Mohd, et al. "A Review of Different Applications of Wireless Sensor Network (WSN) in Monitoring Rehabilitation." Journal of Telecommunication, Electronic and Computer Engineering (JTEC) 10.1-9 (2018): 121-127.

15. Yiu-Wan, Y. I. P., et al. "Biofeedback System with Body Mapping Clothing for Patients with Adolescent Idiopathic Scoliosis." U.S Patent Application No. 15/947,897.

16. Mallick, Arpita, et al. "Energy Efficient Routing Protocol for Ambient Assisted Living Environment." Wireless Personal Communications 109.2 (2019): 1333-1355.

17. Rieger, Stefan. "Ambient assisted living." Die Enden des Körpers. Springer VS, Wiesbaden, 2019. 145-159.

18. Akyildiz, Ian F., et al. "Wireless sensor networks: a survey." Computer networks 38.4 (2002): 393-422.

19. Hossain, Tasmin, et al. "An IDS investigation of the flow field physics within compressible boundary layers." AIP Conference Proceedings. Vol. 2062. No. 1. AIP Publishing, 2019.

20. Chaabouni, Nadia, et al. "Network Intrusion Detection for IoT Security based on Learning Techniques." IEEE Communications Surveys \& Tutorials (2019)

21. Almahdi, E. M., et al. "Mobile patient monitoring systems from a benchmarking aspect: Challenges, open issues and recommended solutions." Journal of medical systems 43.7 (2019): 207.

22. de Carvalho, Carlos Giovanni Nunes, et al. "Multiple linear regression to improve prediction accuracy in WSN data reduction." 2011 7th Latin American Network Operations and Management Symposium. IEEE, 2011.

23. Le Borgne, Y.-A., Raybaud, S., and Bontempi, G. (2008). Distributed Principal Component Analysis for Wireless Sensor Networks. Sensors, 8(8), 4821- 4850

24. Xie, M., Hu, J, Han, S., \& Chen, H. H. (2012). Scalable hypergrid k-NN-based online anomaly detection in wireless sensor networks. IEEE Transactions on Parallel and Distributed Systems, 24(8), 1661-1670.

25. Xie, M., Han, S., Tian, B., and Parvin, S. (2011b). Anomaly detection in wireless sensor networks: A survey. Journal of Network and Computer Applications, 34(4), 1302-1325.

26. Zhang, Y., Meratnia, N., and Havinga, P. J. (2010b). Ensuring high sensor data quality through use of online outlier detection techniques. International Journal of Sensor Networks, 7(3), 141-151

27. Moshtaghi, M., Bezdek, J. C., Havens, T. C., Leckie, C., Karunasekera, S., Rajasegarar, S., et al. (2012). Streaming analysis in wireless sensor networks Wireless.

28. Moshtaghi, M., Havens, T. C., Bezdek, J. C., Park, L., Leckie, C.Rajasegarar, S., et al. (2011a). Clustering ellipses for anomaly detection. Pattern Recognition, 44(1), 55-69.

29. Zhang, Y., Meratnia, N., and Havinga, P. J. (2010b). Ensuring high sensor data quality through use of online outlier detection techniques. International Journal of Sensor Networks, 7(3), 141-151

\section{AUTHORS PROFILE}

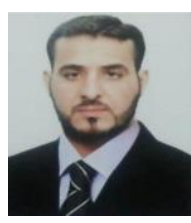

Hayder Jassim Hassab Allah, master student in College of Electrical Engineering Techniques, Middle Technical University, Baghdad, Iraq.

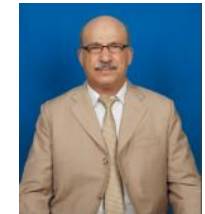

Assoc. Prof. Dr. Rashid Ali Fayadh, received his B.Sc. degree from Middlesex University /Engineering College / UK (London), in 1986 and worked as an electronic warfare engineer in Iraqi air force up to 1998 He received the M.Sc. degree from University of Technology/ Al-Rasheed College / Baghdad-Iraq in 2000 and received the Ph.D degree from University Malaysia Perlis, Malaysia, Perlis

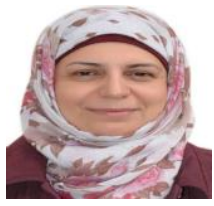

Bushra Abdulkareem Abdulazeez Al- Hayal, Lecturer in: Middle Technical University / Institute of Medical Technology Baghdad / Iraq 